\title{
More than half of ESBL-E are susceptible to fluoroquinolones: admission prevalence data from eight non-ICUs in a German university hospital
}

\author{
F Maechler", N Thoma, P Dem, A Kola, S Hansen, P Gastmeier, R-GNOSIS study group \\ From 3rd International Conference on Prevention and Infection Control (ICPIC 2015) \\ Geneva, Switzerland. 16-19 June 2015
}

\section{Introduction}

Little information is available on prevalence of extendedspectrum-betalactamase-producing Enterobacteriaceae (ESBL-E) with and without additional resistance to fluoroquinolones. In Germany, Enterobacteriaceae are classified according to their susceptibility to four classes of antimicrobial substances. Only organisms resistant to acylureidopenicillins, $3^{\text {rd }}$ and $4^{\text {th }}$ generation cephalosporins and fluoroquinolones are labelled as "multidrug-resistant".

\section{Objectives}

The aim of this prospective analysis was to gain evidence on admission prevalence and incidence of ESBL-E with and without resistance to fluorochinolones in non-ICUs in a German university hospital.

\section{Methods}

This analysis is part of the R-GNOSIS framework. WP 5 investigates the benefits of isolation precautions over standard measures for ESBL-E-carriers in non-ICUs. Rectal swabs are obtained for all patients admitted to the participating wards within 3 days of admission. Patients staying longer than 3 days are screened every 7 days thereafter and before discharge. Chromogenic culture media are used for ESBL-screening, identification and susceptibility testing is performed using Vitek 2 (bioMérieux, Germany).

\section{Results}

Between February 2014 and February 2015, 8317 patients were admitted to 8 medical and surgical wards. An admission sample was obtained for 6047 patients (73\%). Among all 8317 patients, 6814 patients had a LOS of more than 3 days, and 4083 patients were screened at least twice (60\%).

The majority of ESBL-E-carriers was identified on admission ( $\mathrm{n}=607,10.1 \%)$. However, 197 patients (4.8\%) were screened negative on admission and turned ESBLpositive during their stay. Admission prevalence of ESBL-E resistant to fluorochinolones was $4.7 \%(n=286)$, and $2.7 \%(\mathrm{n}=111)$ of patients turned positive during their stay.

\section{Conclusion}

More than 50\% of ESBL-E were susceptible to fluorochinolones. As the increase of ESBL-E is a worldwide concern and resources to prevent their spread are limited, focusing on ESBL-E according to their antimicrobial susceptibility pattern may be a pragmatic approach.

\section{Disclosure of interest}

None declared.

Published: 16 June 2015

doi:10.1186/2047-2994-4-S1-P124

Cite this article as: Maechler et al:: More than half of ESBL-E are susceptible to fluoroquinolones: admission prevalence data from eight non-ICUs in a German university hospital. Antimicrobial Resistance and Infection Control 2015 4(Suppl 1):P124.

Institute of Hygiene, Charité, Berlin, Germany 